\title{
Conditioned Preference to a Methamphetamine-Associated Contextual Cue in Humans
}

\author{
Leah M Mayo', Diana Fraser ${ }^{2,3}$, Emma Childs', Reza Momenan ${ }^{2}$, Daniel W Hommer ${ }^{2}$, Harriet de Wit ${ }^{1,4}$ and \\ Markus Heilig*,2,4
}

'Human Behavioral Pharmacology Laboratory, Department of Psychiatry and Behavioral Neuroscience, MC 3077, The University of Chicago, Chicago, IL, USA; '2 Laboratory of Clinical and Translational Studies, National Institute on Alcohol Abuse and Alcoholism, National Institutes of Health, Bethesda, MD, USA; ${ }^{3}$ Department of Clinical and Experimental Medicine, Linköping University, Liköping, Sweden

\begin{abstract}
Classical conditioning is widely used to study motivational properties of addictive drugs in animals, but has rarely been used in humans. We established a procedure suitable for studying the neurobiology and individual determinants of classical conditioning in humans. Healthy volunteers were randomly assigned to four groups that received methamphetamine or placebo in the presence of distinctive environmental cues under paired or unpaired conditions. During each session, subjects performed tasks known to activate the ventral striatum. Tasks were performed in the presence of a distinctive context, consisting of a screen background image of a beach or mountains, accompanied by corresponding sounds. Separate groups of subjects carried out the tasks under high (\$35-50) or low (\$5-20) reward conditions. Within each of the two reward conditions, one group (paired) received methamphetamine (20 mg, oral) or placebo consistently associated with one of the contexts, while the other (unpaired) received drug or placebo unrelated to context. A fifth group (paired) performed the tasks with contextual cues but in the absence of monetary incentives. Before and after conditioning, participants carried out a series of forced choice tasks for the contextual cues, and change of preference over time was analyzed. All paired groups showed a significant increase in preference for the drug-associated context, with a linear trend for increase across the levels of reward. Preference was unrelated to subjective drug effects, and did not change in the unpaired group. These data support the translational utility of our conditioning procedure for studies of reward mechanisms in humans.

Neuropsychopharmacology (2013) 38, 921 -929; doi:I0.1038/npp.2013.3; published online 27 February 2013
\end{abstract}

Keywords: conditioning; place preference; stimulant; amphetamine; reward

\section{INTRODUCTION}

An extensive preclinical literature indicates that environmental stimuli paired with rewarding drugs acquire incentive value through the process of classical conditioning, ie, cues or contexts consistently predictive of a rewarding drug acquire value in and of themselves. This process is often studied in animals using the conditioned place preference (CPP) procedure, in which animals come to prefer an environment in which they have received a drug known to have rewarding effects (Tzschentke, 1998, 2007). Although the process of conditioning, and CPP in particular has been studied in depth in animals, very few studies have investigated the acquisition of conditioning in humans. We recently showed that humans, like other species, acquire preference for a physical environment (room) where they have received a rewarding stimulant drug (Childs and de

*Correspondence: Dr M Heilig, LCTS, NIAAA, 10 Center Drive, I0/IE-5334, Bethesda, MD 20892-I I08, USA. Tel: + I 30I 7687326, Fax: + 30 | 45|7498, E-mail: markus.heilig@mail.nih.gov

${ }^{4}$ These authors contributed equally to this work.

Received II November 2012; revised 13 December 2012; accepted 14 December 2012; accepted article preview online 28 January 2013
Wit, 2009, 2011). The degree of this preference was positively associated with the degree of drug liking, supporting the idea that place preferences are determined by the rewarding properties of the drug. In the present study, we extended our investigation of classically conditioned drug effects in humans, by developing a procedure that relies on virtual rather than physical contextual stimuli. We used a compound environmental stimulus consisting of a computer image with corresponding sounds, paired with drug. We also sought to develop a procedure that, similar to animal studies of CPP, would rely on choice behavior, rather than verbal report of subjective liking of drug-paired context.

Conditioning with drugs as unconditioned stimuli involves several processes. First, the drug may produce feelings of well-being, which confer salience to cues that predict the cue. Our data with CPP in humans support this idea. Second, the drug may facilitate the process of learning. That is, a stimulant drug may enhance learning about environmental stimuli, because of its effects on attention or encoding. Third, the drug may activate dopaminergic (DA) processes involved in learning, and increasing this DA activity may fundamentally affect the associative process. 
Although the present study cannot distinguish between these possibilities, it lays the foundation for future studies investigating the role of DA in acquisition of incentive valence and salience, and ultimately in aberrant learning leading to drug abuse.

Incentive salience of environmental stimuli is thought to be encoded in part by DA activity in the ventral striatum (Robinson and Berridge, 2003). We hypothesized that methamphetamine, which potentiates DA transmission, would confer incentive salience to an initially neutral context, thereby increasing subject preference for it. Because methamphetamine is an indirect DA agonist, we further speculated that it may interact with endogenous ventral striatal activity and potentiate the incentive salience associated with ongoing processing within this structure, as suggested by previous findings with methylphenidate (Volkow et al, 2004). To address this latter hypothesis, we designed a procedure in which subjects were engaged in tasks originally developed for event-related fMRI studies, and shown by these studies to be consistently associated with ventral striatum activation. For the best established of this tasks, the monetary incentive delay (MID) task, ventral striatal activation has also been shown to scale with the magnitude of anticipated monetary reward (Knutson et al, $2000,2001)$. We therefore assigned subjects to groups where the monetary incentive was high $(\$ 35-50)$, low $(\$ 5-20)$, or absent.

To establish our procedure, we used a classical conditioning paradigm in which participants experienced the drug and the environmental stimuli under paired or unpaired conditions. The primary outcome measure was the change in preference for the environmental stimuli after conditioning. Subjects also rated subjective drug effects, and we sought to determine whether these were predictors of preference conditioning. During the procedure, participants performed a series of tasks in the presence of the distinctive visual and auditory stimuli ('context'). They worked for the possibility to earn monetary rewards, which varied across groups. The procedure used a stimulus presentation environment designed for use in an fMRI environment, providing the means for future studies addressing the determinants, consequences, and underlying neurobiology of conditioned drug effects in humans.

\section{MATERIALS AND METHODS}

\section{Participants}

Healthy volunteers aged 18-35 were recruited through posters, online and newspaper advertisement, and word-ofmouth referrals throughout the community. Inclusion criteria were body mass index (BMI) between 19 and $26 \mathrm{~kg} / \mathrm{m}^{2}$, completed high school education, fluency in English, resting blood pressure (BP) $<140 / 90 \mathrm{~mm} \mathrm{Hg}$, current alcohol consumption $<4$ standard drinks/day, and consumption of $<4$ units of caffeinated beverages per day. Exclusion criteria were current major Axis I DSM-IV disorder (American Psychiatric Association, 2000), including current dependence on, or abuse of alcohol or illicit drugs; past alcohol or drug dependence, or psychotic disorder; diagnosis of a mood disorder within the past year; ongoing treatment with psychoactive medication, cardiovascular illness; or past or present medical conditions contraindicating methamphetamine, shift work, pregnancy, nursing, or plans to become pregnant. Women not on hormonal birth control were scheduled for testing sessions only during their follicular phase (White et al, 2002). Eligible candidates completed the psychiatric symptom checklist (Derogatis, 1983), the Beck Depression Inventory (Beck et al, 1996), the Michigan Alcoholism Screening test (Selzer, 1971), the Barratt Impulsiveness Scale-11 (BIS 11; Patton et al, 1995), and underwent a physical examination and electrocardiogram. Qualifying participants attended one 1-h orientation session to read and sign the consent form, outlining the procedures and listing the classes of drugs and their possible effects. To prevent expectancy effects, participants were informed that they could be given placebo, a stimulant, or a sedative drug, and they were told that the purpose was to investigate the effects of drugs upon mood and performance on tasks. Following completion of the study, participants were fully debriefed. The study took place at the Human Behavioral Pharmacology Laboratory at the University of Chicago Hospital. It was approved by the University of Chicago Biological Science Division Institutional Review Board, and all subjects provided written informed consent. Participants were paid for their participation.

\section{Drugs}

Methamphetamine was selected because of its relative fast onset. The dose used (20 mg oral; Desoxyn, Lundbeck) is safe and produces robust subjective effects (Martin et al, 1971; Cook et al, 1992). To speed the absorption time, the tablets were crushed and administered in $50 \mathrm{ml}$ sugar-free syrup (Ora-Sweet, Paddock Laboratories, Minneapolis, MN). Placebo solutions consisted of $50 \mathrm{ml}$ OraSweet alone. The oral liquid was given in a transparent $50 \mathrm{ml}$ syringe.

\section{Overall Design}

Subjects were assigned to one of five groups: Paired, high reward (PairHi; $n=24$ ); Paired, low reward (PairLo; $n=24$ ); Paired no reward (PairNone; $n=23$ ); Unpaired, high reward (UnpHi; $n=7$ ); and Unpaired, low-reward (UnpLo; $n=9$ ). Each participant attended an orientation session, followed by four 4-h conditioning sessions separated by at least $48 \mathrm{~h}$, and a 2 -h test session. During the initial orientation session, subjects practiced the computer tasks together with the stimuli to be paired during the conditioning sessions, and carried out a series of choices for the tasks and images (see below). This provided a pre-conditioning measure of their preference for the respective stimuli, which was then used to assess changes after the pairings. All subjects received methamphetamine $(20 \mathrm{mg})$ on two conditioning sessions and placebo on the other two sessions, administered under double-blind conditions. Subjects differed in whether environmental stimuli were consistently paired with the drug administration. The three paired groups consistently received drug and placebo in combination with one of the two sets of visual and auditory background stimuli (see below). The two unpaired groups received each treatment in 
combination with one of the stimuli sets on one session, and the other on the other (Figure 1).

During all four conditioning sessions, subjects performed a series of computer tasks known to activate the ventral striatum in event-related fMRI studies. To maintain interest, three different tasks were used: a version of the previously published MID task (Knutson et al, 2000, 2001), a Risk (R) task (Gilman et al, 2012), and a Prediction Error (PE) task. Depending on group assignment, subjects were able to earn high (\$35-50/session; PairHi, UnpHi) or low monetary reward (\$5-20/session, PairLo, UnpLo), but this was not contingent on subjects' performance. Each run for each game began with a $\$ 5.00$ credit to prevent a negative outcome early in the run. The PairNone group played the same games without monetary reward. The order of the three games and the background images used were randomized and counterbalanced across subjects, but remained constant within subjects.

Tasks were presented in the presence of the two distinctive compound stimuli consisting of a background screen visible behind the task presentation and accompanied by a corresponding sound. One set of stimuli depicted a summer beach view accompanied by the sound of ocean waves. The other depicted summer alpine scenery accompanied by birdsong. Presentation software (Neurobehavioral Systems, San Francisco, CA) was used for delivery and control of both visual and audio stimuli.

\section{MID Task}

The modified MID task consisted of 32 trials each lasting about $12 \mathrm{~s}$. A trial consisted of a 1.5-s presentation of a cue shape, followed by a fixation crosshair of a variable duration (1.3-4.0 s). A white target box was then displayed for $0.5-0.6 \mathrm{~s}$, signaling subjects to press a button. After another variable interval (1.3-4.0 s), subjects were given feedback for $1.5 \mathrm{~s}$ about the amount of money they had won or lost during that trial, as well as their running total. Trials were separated by 1.3-4.0 s. Participants could win or avoid losing money by pressing a button during target presentation. Cues signaled potential reward $(n=20$; denoted by circles), potential loss prevention $(n=9$; denoted by squares), or no monetary outcome $(n=3$; denoted by triangles). In the high reward groups, a circle with one line signaled the potential to win $\$ 0.25$, and a circle with three lines $\$ 1.00$; in the low reward groups, these values were $\$ 0.1$ and $\$ 0.5$. The square cues were loss prevention signals. If the participant did not press the button in time for when the target box appeared, then they lost the amount the cue symbolized, $\$ 0.25$ for a square with one line and $\$ 1.00$ for a square with three lines $(\$ 0.25$ and 0.50 for low earning group). Trial types were pseudo randomly ordered within each session and the task lasted $\sim 6.5 \mathrm{~min}$.

\section{Risk Task}

This is a version of the Lane risk-taking task (Lane and Cherek, 2000), as modified in Gilman et al (2012). Briefly, each trial consisted of displaying, for $1.5 \mathrm{~s}$, two white squares, one with a question mark beneath it. The participant was forced to choose between these two response options. The square with the question mark (the risky choice) gave the possibility to win or lose $\$ 0.50$ or $\$ 1.00$, or win or lose $\$ 0.25$ or $\$ 0.10$, in the high and low earning groups, respectively. The square without the question mark beneath it (safe choice) guaranteed the participant to win $\$ 0.50$ or $\$ 0.05$ for the high $v s$ the low earning groups. Participants made their choice by pressing a mouse button corresponding to the left or the right square and the selected square re-appeared on the screen for $1.5 \mathrm{~s}$. The participant was then shown a screen with feedback of how much money was lost or won for that trial, as well as the running total for $1.5 \mathrm{~s}$. A crosshair appeared for a variable interval of (1.3-4.0 s) between the choice event and feedback as well as between trials. Each risk task consisted of 36 trials, each lasting on average $11.2 \mathrm{~s}$, yielding a total of $\sim 6.9$ min.

\section{PE Task}

The PE task consisted of simultaneously presenting to the participant with two different abstract images on the computer screen $(1.5 \mathrm{~s})$. Each set of simultaneously presented images was associated with a predefined probability of winning or losing. Choosing one of the images was associated with a probability of 80 vs 20\% for winning or

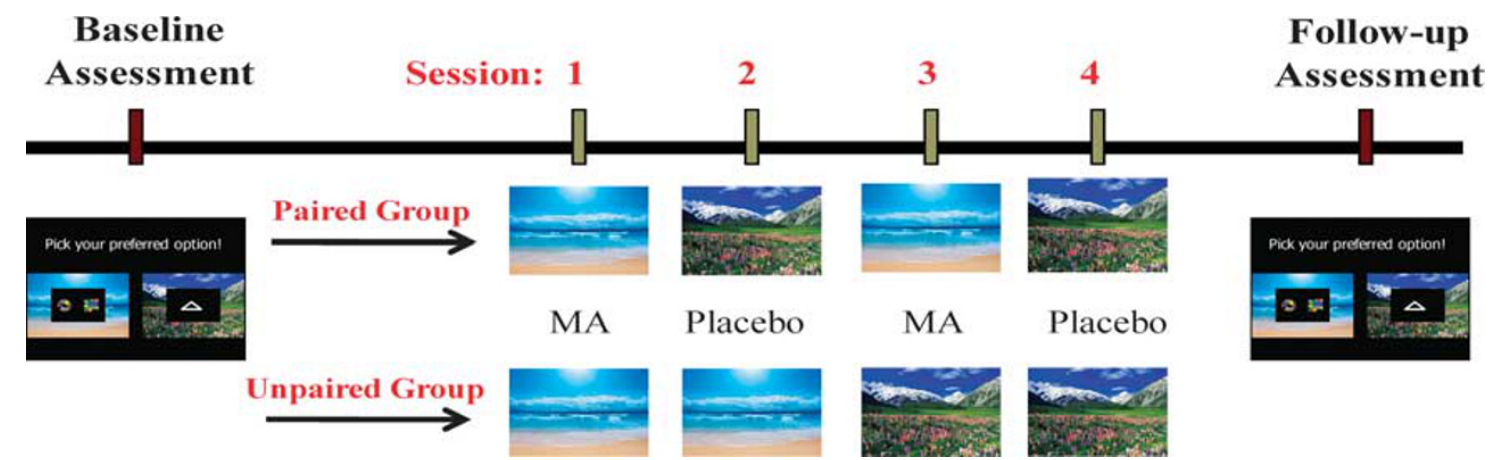

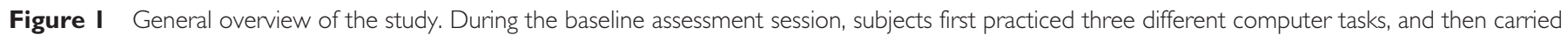

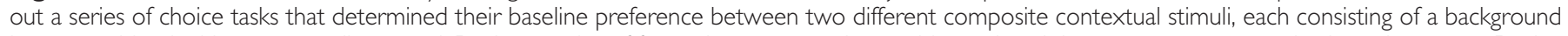

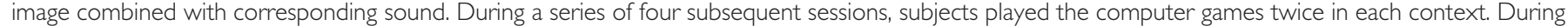

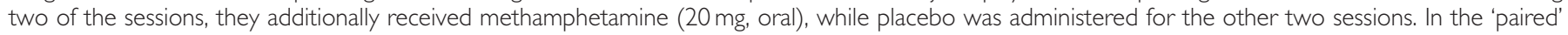

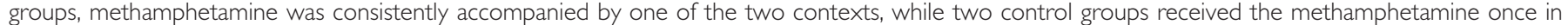
each context. 
losing, respectively; choosing the other image was associated with the opposite probabilities. The position as well as the image associated with the respective outcome set was both randomized and counterbalanced. The potential earnings were $\$ 0.75$ and $\$ 1.00$ in the low and high reward groups, respectively. The potential loss was $\$ 0.50$ and $\$ 0.25$ in the low and reward groups, respectively. During the game, the winning and losing images could switch (task reversal). A white square around the participant's choice informed them that their selection was detected $(1.5 \mathrm{~s})$. Next, after a random interval (1.3-4.0 s), a feedback was shown for $1.5 \mathrm{~s}$ indicating the amount of earning or loss in the trial, as well the running total. Between trials, a crosshair was seen for a variable interval (1.4-4.0 s). Each PE task consisted of 36 trials, each lasting on average $11.0 \mathrm{~s}$. Participants played one run of $\sim 6.8 \mathrm{~min}$. Subjects were instructed verbally by the experimenter before the task.

\section{Rating Task}

Subjects completed a task to assess their preferences for the contextual stimuli before and after conditioning. They were asked to choose between pairs of stimuli consisting of both the tasks and the backgrounds, across 15 separate trials, in a full-factorial design. Assessment of task preferences (ie, choices between tasks with the same background) was included only to rule out preference biases between tasks at baseline or after conditioning. Because no task bias was observed at either time point, we collapsed the tasks for analysis. This left nine trials that provided information about the background preferences. Our primary outcome measure was the change in background preference from before to after conditioning. On each trial subjects chose between two images, of one of the three tasks combined with one of the two backgrounds. All the three tasks were combined with each of the two backgrounds ( 15 trials). The two images were first presented separately for $3 \mathrm{~s}$ each, and then shown side-by-side for the choice phase (Figure 1). Subjects indicated their preferred image by pressing the corresponding mouse button.

\section{Subjective and Physiological Measures}

During each of the conditioning sessions, BP and heart rate (HR) were repeatedly measured, and participants completed self-report computer-based, standardized subjective mood and drug effects questionnaires. All measures were obtained at $-15,+30,+70,+115$, and +210 min relative to study drug administration. The subjective measures were Profile of Mood States (POMS; McNair et al, 1971); Addiction Research Center Inventory (ARCI; Haertzen, 1966), and the Drug Effects Questionnaire (DEQ; Johanson and Uhlenhuth, 1980). At the end of each of the conditioning sessions, an end-of-session questionnaire (ESQ) was completed, designed to retrospectively assess subjective drug effects. This included: liking of drug effects, assessed using a $100-\mathrm{mm}$ visual analog scale (VAS) ranging from 'dislike' to 'like very much'; overall assessment of subjective drug effects, given as a choice between five statements: (1) no effect, (2) a mild effect, but I'm not sure, (3) definitely an effect, but it was not strong, (4) a strong effect, and (5) a very strong effect; what drug do you think the oral solution contained by choosing one of four statements (a) stimulant/appetite suppressant, (b) sedative/tranquilizer, (c) alcohol, and (d) placebo. Finally, participants responded to a question whether they would take the oral solution again if given the opportunity.

\section{Session Procedures}

Subjects were instructed to consume their normal amount of caffeine/nicotine before sessions but to abstain from using prescription and over-the-counter drugs $24 \mathrm{~h}$ before and $6 \mathrm{~h}$ after each session; other recreational drugs for 2 days before and $6 \mathrm{~h}$ after sessions; marijuana for 7 days before and $6 \mathrm{~h}$ after each session; and to not operate any machinery or vehicles for $6 \mathrm{~h}$ following each session. Compliance measures were obtained before all sessions, and included a self-report questionnaire, breath alcohol levels (Alco-SensorIII, Intoximeters, St Louis, MO), urine drug test (ToxCup, Branan Medical Corporation, Irvine, CA) and for women a pregnancy test (AimStickPBD, hCG professional, Craig Medical distribution, Vista, CA). During the orientation, participants practiced MID, PE, and Risk tasks ( $2 \mathrm{~min} /$ task) and questionnaires. They also performed one complete run of the rating task.

Sessions were conducted from 0900 to $1300 \mathrm{~h}$, with at least $48 \mathrm{~h}$ between sessions. After compliance tests (BAL, urine toxicology and pregnancy), pre-drug measures were obtained and subjects received the drug. Conditioning sessions and the rating task session were performed individually in the same room for each participant, which was different from the room in which the orientation session took place. The rooms were comfortably furnished with a couch, a desk and chair, a computer (for tasks and questionnaires), a television, a video player, and magazines. When participants were not completing tasks, questionnaires or having their BP and HR measured, they were allowed to relax, watch selected movies or read. Participants were not allowed to sleep, work, or study. Cellphones or Internet access was not allowed, minimizing emotional contact outside the testing environment. Tasks were performed between +30 and +70 min after study drug administration, timed to coincide with peak plasma concentration of methamphetamine (Cook et al, 1992). Participants completed an end-of-study questionnaire when other measures were done. They were allowed to leave once subjective and cardiovascular drug effects subsided. The final rating session was conducted at least $48 \mathrm{~h}$ after the last conditioning session. On this session, subjects provided compliance measures (urine, breath alcohol), completed baseline questionnaires and then completed the postconditioning Rating task.

\section{Data Analysis}

Subjective and physiological treatment effects were analyzed using mixed model ANOVA's, with within-session time as within-subjects factors, and treatment (MA, placebo) as between-subjects factors. Differences at individual time points were evaluated using Tukey's post-hoc test. The primary outcome analysis was the change in preference for the three paired groups, using a mixed model ANOVA with phase (pre- and post-conditioning) as within-subjects 
factor, and reward condition (high, low, none) as a betweensubjects factor. Baseline variables as well as subjective drug effects were evaluated for contribution as co-variates in the analyses. Because of its range and distributions, lifetime drug use was dichotomized, and was among the baseline measures evaluated as co-variates. Co-variates were dropped if they did not contribute significantly to the respective model, or reduced its residual variance.

\section{RESULTS}

\section{Baseline Characteristics}

Baseline characteristics of the combined sample and the five experimental groups are shown in Table 1. Groups did not differ except for variables indicated. To control for any group differences, the potential contribution of baseline variables to the outcome analyses was evaluated, and is indicated below where present.

\section{Subjective and Physiological Responses}

Methamphetamine increased ratings on the ARCI amphetamine scale (main drug effect: $F(1,86)=284.01, P<0.001$, main time effect: $F(5,430)=77.87, P<0.0001$, drug $\times$ time interaction: $F(5,430)=56.37, P<0.0001$; Figure 2a). Post hoc tests showed that the drug effect was present already after $15 \mathrm{~min}$, peaked between 30 and $70 \mathrm{~min}$, coinciding with the conditioning task, and remained significantly higher throughout the session. There were no significant covariates in the model.

Methamphetamine also increased ratings of drug liking on the DEQ (main drug effect: $F(1,85)=57.12, P<0.001$, main time effect: $F(5,425)=26.25, P<0.0001$, drug $\times$ time interaction: $F(5,425)=16.77, P<0.0001$; Figure $2 \mathrm{~b})$. Post hoc tests showed that the drug effect was present already after $15 \mathrm{~min}$, and peaked between 30 and $70 \mathrm{~min}$, coinciding with the conditioning task. The number of servings of caffeine in the previous month was a significant co-variate in this model. A sensitivity analysis showed that the interaction effect of the experimental factors remained unchanged in the absence of this co-variate.

Methamphetamine increased mean arterial BP (main drug effect: $F(1,84)=206.04, P<0.0001$, main time effect: $(F(5,420)=56.87, P<0.0001$, treatment $\times$ time interaction: $F(5,420)=96.76, P<0.0001$ on mean arterial pressure; Figure 2c). There was also a main effect of sex on mean arterial pressure, with males $($ mean $=94.65, \mathrm{SEM}=1.02)$ having higher $\mathrm{BP}$, on average, than females (mean $=89.89$, $\mathrm{SEM}=0.90$ ).

There were no main effects of drug treatment $(F(1,82)$ $=1.45, P=0.23)$ or time $(F(5,410)=0.41, P=0.84)$ on $\mathrm{HR}$; however, there was a drug treatment-by-time interaction $(F(5,410)=3.13, P=0.009)$, indicating that the two groups were differentially influenced by drug treatment. Specifically, HR decreased over the course of sessions under placebo treatment, but remained relatively unchanged under drug treatment. Significant co-variates in the model for HR included BMI, average number of drinks per day, and the maximum number of drinks per day in the past month. A sensitivity analysis showed that the interaction effect of the experimental factors remained unchanged in the absence of these co-variates. Similarly to BP, HR was elevated during the drug session compared with the placebo session (Figure 2d).

\section{Preference Ratings}

For the paired groups, preference for the stimuli increased from before to after conditioning $(F(1,68)=9.74$, $P=0.003)$. Preference ratings were significantly higher in the post test phase, irrespective of reward condition (Figure 3). The rank order of mean preference scores was high reward $>$ low reward $>$ no reward, but reward condition was not a significant co-variate in the analysis, presumably due to variability in the data $(F(2,68)=0.61$, $P=0.54)$. No baseline characteristics were significant covariates in the model for the paired group.

An interim analysis of the unpaired groups showed that preference did not change as a function of test phase $(F(1,14)=0.6, P=0.81)$ or reward condition $(F(1,14)$ $=0.53, P=0.48)$, nor was there any interaction effect between these two factors $(F(1,14)=0.36, P=0.56)$. As with the paired groups, there were no significant co-variates in the model for the unpaired group. Because this interim analysis showed that either no effect of the experimental factors was present, or, if present, its size would have to be so small that it would require excessive number of subjects to be detected, these arms filled criteria for futility, and recruitment into them was terminated.

\section{Correlations Between Subjective Effects and Preference}

We examined individual differences in liking of the drug (DEQ) in relation to the change in preference for the background stimuli (ie, conditioning) in the paired groups. A scatterplot of the change in preference score from pre- to post-conditioning $v s$ subjective drug liking as assessed by the DEQ (Figure 4) shows that there was no correlation between the two measures in any of the reward conditions.

\section{DISCUSSION}

We demonstrated that healthy young adults develop a robust preference for a virtual contextual stimulus paired with a moderate dose of methamphetamine. That is, an initially neutral compound stimulus acquired incentive salience, or positive motivational properties, through its association with a rewarding drug. The study used the procedures of classical conditioning, parallel to procedures used in laboratory animals, and closely resembling other recent studies with d-amphetamine in humans (Childs and de Wit, 2009, 2011). A stringent control for the specificity of the conditioning effect was provided by inclusion of nonpaired control groups, which received the same doses of methamphetamine and the same stimuli, but these were not systematically paired. In these groups, change in preference was close to zero, showing that the change in preference observed in the paired groups was not simply related to exposure to either the drug or the stimuli. The study provides a proof of principle that a drug with an ability to increase synaptic DA effectively sustains learning, and confers salience on otherwise neutral stimuli in humans. 


\section{Contextual conditioning to methamphetamine in humans}

LM Mayo et al

Table I Subject Characteristics by Group

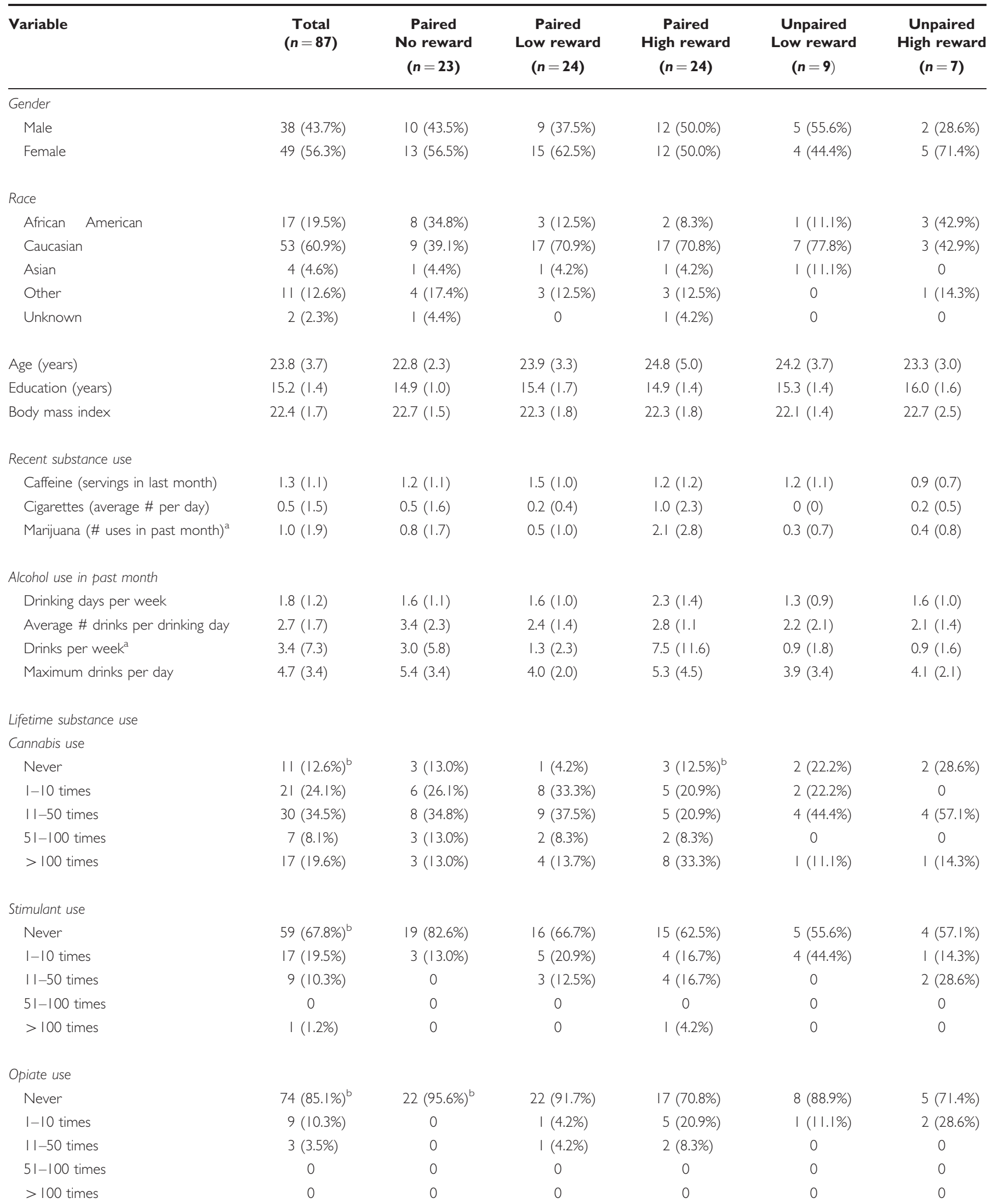


Table I (Continued)

\begin{tabular}{|c|c|c|c|c|c|c|}
\hline Variable & $\begin{array}{c}\text { Total } \\
(n=87)\end{array}$ & $\begin{array}{c}\text { Paired } \\
\text { No reward } \\
(n=23)\end{array}$ & $\begin{array}{c}\text { Paired } \\
\text { Low reward } \\
(n=24)\end{array}$ & $\begin{array}{l}\text { Paired } \\
\text { High reward } \\
(n=24)\end{array}$ & $\begin{array}{l}\text { Unpaired } \\
\text { Low reward } \\
(n=9)\end{array}$ & $\begin{array}{l}\text { Unpaired } \\
\text { High reward } \\
\quad(n=7)\end{array}$ \\
\hline Never & $56(64.4 \%)^{b}$ & $17(74.9 \%)^{b}$ & 15 (62.5\%) & $14(58.3 \%)^{b}$ & $6(66.7 \%)$ & $4(57.1 \%)$ \\
\hline $1-10$ times & $23(26.4 \%)$ & $5(21.7 \%)$ & $6(25.0 \%)$ & 7 (29.2\%) & $2(22.2 \%)$ & $3(42.9 \%)$ \\
\hline$>100$ times & । (1.2\%) & 0 & 0 & I (4.2\%) & & 0 \\
\hline \multicolumn{7}{|l|}{ MDMA use } \\
\hline Never & $62(71.3 \%)^{b}$ & 17 (73.9\%) & $18(75.0 \%)$ & $15(62.3 \%)^{b}$ & 7 (77.8\%) & 5 (71.4\%) \\
\hline $1-10$ times & $23(26.4 \%)$ & $6(26.1 \%)$ & $6(25.0 \%)$ & 8 (33.3\%) & 2 (22.2\%) & I (I4.3\%) \\
\hline
\end{tabular}

${ }^{a}$ Significant group effect (ANOVA, $\left.P<0.05\right)$. For both drinks per week and uses of marijuana in the past month, the Paired/High Reward group is significantly different from the Paired/Low Reward group.

${ }^{b}$ Data missing for one participant.

a

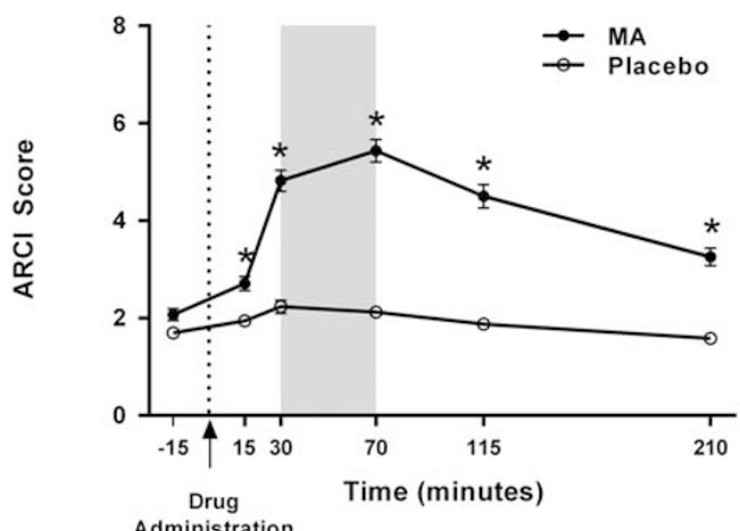

C

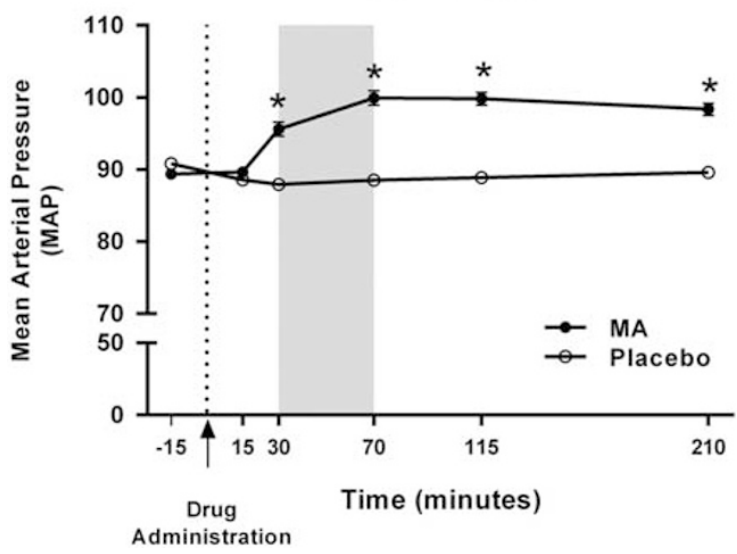

b

DEQ Drug Liking

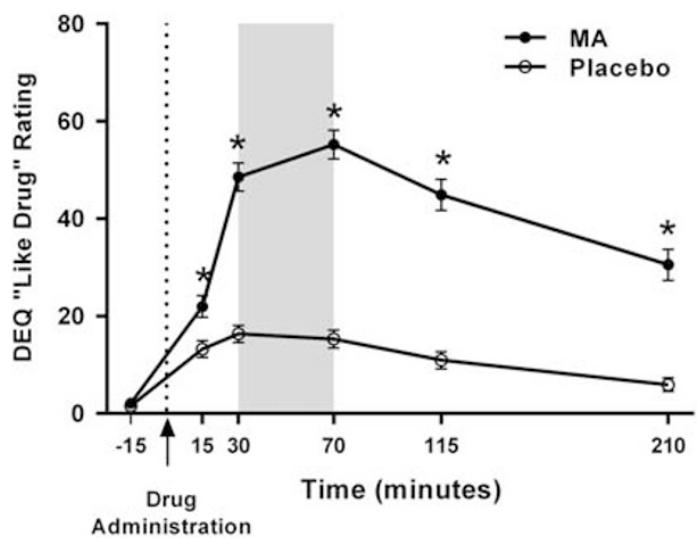

d

Heart Rate

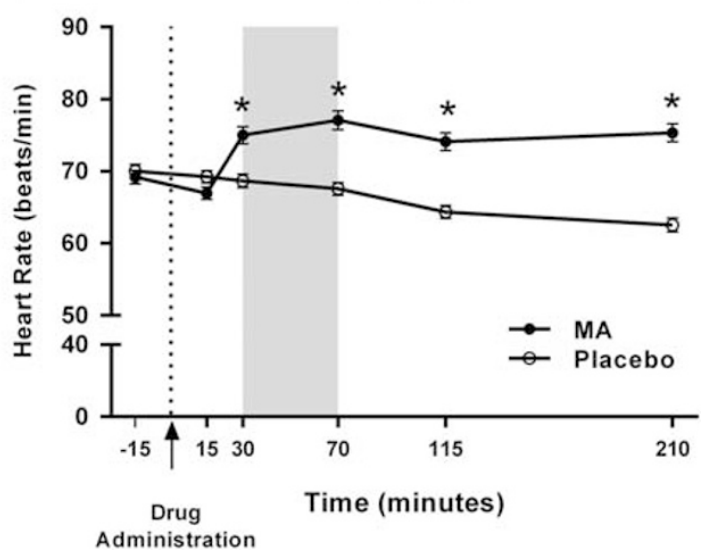

Figure 2 Subjective (a, b) and physiological (c, d) responses to drug and placebo. Arrows indicate drug administration. Indicated in gray in each graph is the interval during which subjects played the computer games, presented together with the respective contextual stimulus ('conditioning'). It can be seen that conditioning coincided with maximal subjectively pleasurable effects of methamphetamine. Means of the two drug and placebo sessions, respectively, are presented, collapsed across both paired and unpaired groups. Data are mean $\pm \mathrm{SEM}$; $* P<0.05$ the respective time point. For detailed statistics, see Results. 


\section{Paired Groups}

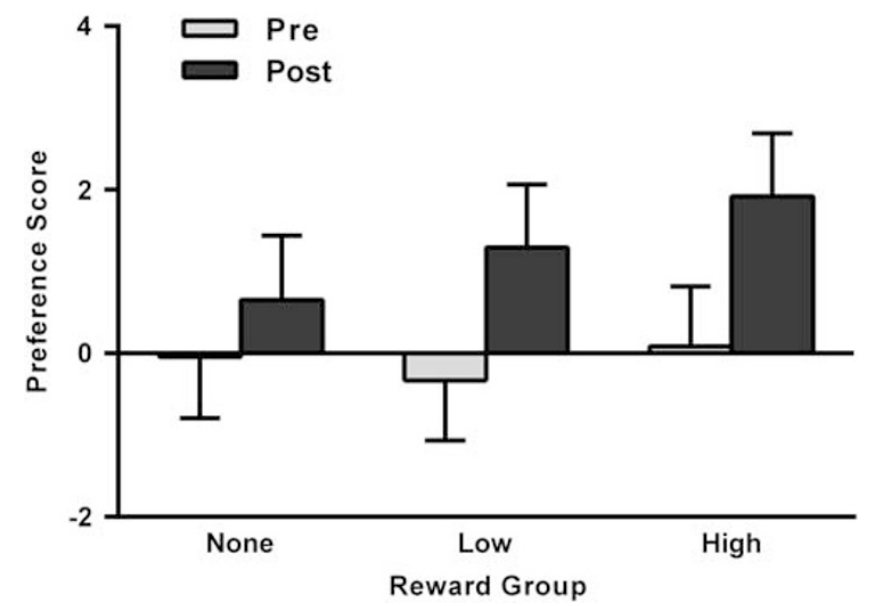

Figure 3 Preference for drug-associated context prior (gray) and after (black) the series of four conditioning sessions. Preference was assessed using a series of nine forced choice trials. A highly significant increase in preference was present across groups that were rewarded at different levels when they carried out the computer tasks during conditioning $(P=0.003$, for detailed statistics, see Results). The rank order of increase in preference followed reward magnitude. Data are mean \pm SEM. No increase in preference was observed in two non-paired control groups (for data, see Results).

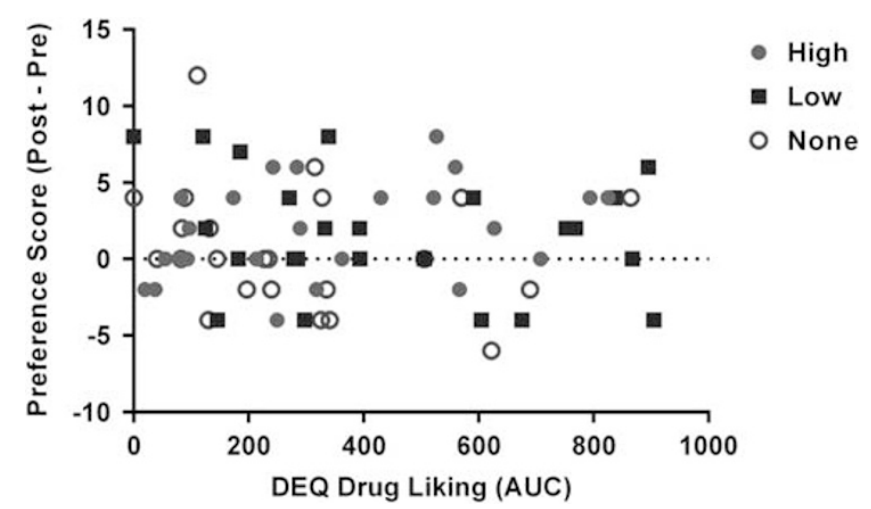

Figure 4 Relationship between subjectively reported drug liking, assessed using the Drug Effects Questionnaire, and preference for drugassociated context, assessed as indicated in Figure 3. Each symbol indicates an individual subject. Each symbol category represents the reward condition (None, Low, or High) under which conditioning was carried out. Irrespective of reward condition, there was no systematic relationship between subjective drug liking and conditioned contextual preference.

The study included some features that help shed light on processes contributing to the drug-cue association. First, the participants reported their subjective state, or liking of the drug during the conditioning trials, allowing us to examine the relation between drug-induced euphoria and effective conditioning. Contrary to expectations based on our previous studies (Childs and de Wit, 2009, 2011), the increase in preference for a drug-associated stimulus in the present study was not correlated with the degree of euphoria experienced. An interesting possibility is that this difference is due to the different methodology used to assess preference. The present study used a fast paced behavioral choice task that may more closely resemble procedures used when contextual preference is assessed in experimental animals. In contrast, the initial studies used verbal report of 'liking' explicitly attributed by subjects to the respective context. It is well established that multiple processes influence explicit report of mental content (Nisbett and Wilson, 1977; Wilson and Dunn, 2004). In addition, it might be speculated that the difference between the two outcome measures reflects a postulated dissociation between 'wanting', as expressed in approach behavior, and 'liking', experienced as a subjective state (Robinson and Berridge, 2003). At a minimum, our data show that conditioning with stimulant drugs in humans is not fully dependent on consciously experienced and explicitly reported feelings of well-being produced by the drug.

Second, we assessed the magnitude of increases in preference as a function of monetary reward level while subjects carried out tasks known to activate the ventral striatum (Knutson et al, 2000, 2001; Gilman et al, 2012). We hypothesized that methamphetamine may confer incentive salience to initially neutral stimuli by potentiating ventral striatum activity, which in the MID task scales with the magnitude of monetary reward. We therefore varied the amount of money subjects received while they were exposed to the conditioned stimulus, from no money, to a low amount or a high amount. Although the level of monetary reward was not a significant factor in the statistical analysis, there was a consistent rank order for the preference scores, high $>$ low $>$ none. This suggests that our conditioning paradigm may become useful for assessment of drug conditioning as a function of reward magnitude, provided that the methodology can in the future be refined to decrease variability in the data. It should be noted that subjects received money in BOTH the drug-paired and the placebopaired environment, so any facilitated conditioning would have to represent an interaction between drug and money.

Third, because standard fMRI tasks and stimulus presentation environment were used, our data suggest a utility of this model to assess neural activity underlying drug conditioning in future studies. Specifically, it will be important to determine whether similar brain circuits are activated by stimuli associated with rewards, and the areas primarily activated by the rewards themselves. Also, it will be of interest to determine whether drugs with rewarding properties further enhance brain responses to the conditioned stimuli. Finally, it will be possible to study individual determinants of drug conditioning, including genetic factors. For instance, we have recently shown that DA release within ventral striatum in response to an alcohol challenge is moderated by mu-opioid receptor gene variation (Ramchandani et al, 2011), but potential implications of this for acquisition of drug associations remain unknown. Meanwhile, others have shown differential DA response to drugs between men and women (see eg, Urban et al, 2010).

In summary, we demonstrate robust conditioning of a stimulant drug to an initially neutral virtual context. As noted in Introduction, a stimulant drug may influence the development of conditioning through any of several processes. Our findings do not support the idea that the development of conditioning is related simply to the feelings of well-being produced by the drug, because there was no correlation between subjects' ratings of 'drug liking' 
and change in preference for the conditioned stimulus. It is possible that the methamphetamine enhanced the salience of the contextual stimulus, perhaps by facilitating the process of learning through its effects on attention or encoding. A more intriguing mechanistic explanation of the facilitation is that methamphetamine, and perhaps also the concurrent receipt of money, activate DA processes that are integrally involved in learning. This DA activity may facilitate, or indeed even underlie, the associative process.

\section{ACKNOWLEDGEMENTS}

This research was supported by the Division of Intramural Clinical and Biological Research NIAAA (MH) and by DA02812 (HdW). Leah Mayo was supported by T32 DA007255. We want to thank Dr Melanie Schwandt for data analysis and Mrs Karen Smith for assistance with the bibliography.

\section{DISCLOSURE}

HdW has received support from Unilever for a project unrelated to this research. $\mathrm{MH}$ is or has been the PI on Cooperative Research and Development Agreements between the NIH and Eli Lilly, Johnson \& Johnson, Merck, Bristol Meyer Squibb, and GlaxoSmithKline unrelated to this project.

\section{REFERENCES}

American Psychiatric Association (2000). Diagnostic Criteria from $D S M-I V-T R$. American Psychiatric Association: Washington, DCxii: 370 pp.

Beck AT, Steer RA, Brown GK (1996). Manual for the Beck Depression Inventory-II. Psychological CorporationSan Antonio, TXVol 1: $82 \mathrm{pp}$.

Childs E, de Wit H (2009). Amphetamine-induced place preference in humans. Biol Psychiatry 65: 900-904.

Childs E, de Wit H (2011). Contextual conditioning enhances the psychostimulant and incentive properties of d-amphetamine in humans. Addict Biol, e-pub ahead of print, 29 November 2012, doi:10.1111/j.1369-1600.2011.00416.x.

Cook CE, Jeffcoat AR, Sadler BM, Hill JM, Voyksner RD, Pugh DE et al (1992). Pharmacokinetics of oral methamphetamine and effects of repeated daily dosing in humans. Drug Metab Dispos 20: $856-862$.

Derogatis L (1983). SCL-90-R: Manual-II. Clinical Psychmetric Research: Towson, MD.

Gilman JM, Smith AR, Ramchandani VA, Momenan R, Hommer DW (2012). The effect of intravenous alcohol on the neural correlates of risky decision making in healthy social drinkers. Addict Biol 17: 465-478.
Haertzen CA (1966). Development of scales based on patterns of drug effects, using the addiction Research Center Inventory (ARCI). Psychol Rep 18: 163-194.

Johanson CE, Uhlenhuth EH (1980). Drug preference and mood in humans: Diazepam. Psychopharmacology (Berl) 71: 269-273.

Knutson B, Adams CM, Fong GW, Hommer D (2001). Anticipation of increasing monetary reward selectively recruits nucleus accumbens. J Neurosci 21: RC159.

Knutson B, Westdorp A, Kaiser E, Hommer D (2000). FMRI visualization of brain activity during a monetary incentive delay task. Neuroimage 12: 20-27.

Lane SD, Cherek DR (2000). Analysis of risk taking in adults with a history of high risk behavior. Drug Alcohol Depend 60: 179-187.

Martin WR, Sloan JW, Sapira JD, Jasinski DR (1971). Physiologic, subjective, and behavioral effects of amphetamine, methamphetamine, ephedrine, phenmetrazine, and methylphenidate in man. Clin Pharmacol Ther 12: 245-258.

McNair D, Lorr M, Droppleman L (1971). The Profile of Mood States Manual. Educational and Industrial Testing Service: San Diego, CA.

Nisbett RE, Wilson TD (1977). Telling more than we can know verbal reports on mental processes. Psychol Rev 84: 231-259.

Patton JH, Stanford MS, Barratt ES (1995). Factor structure of the Barratt Impulsiveness Scale. J Clin Psychol 51: 768-774.

Ramchandani VA, Umhau J, Pavon FJ, Ruiz-Velasco V, Margas W, Sun $\mathrm{H}$ et al (2011). A genetic determinant of the striatal dopamine response to alcohol in men. Mol Psychiatry 16: 809-817.

Robinson TE, Berridge KC (2003). Addiction. Annu Rev Psychol 54: 25-53.

Selzer ML (1971). The Michigan alcoholism screening test: the quest for a new diagnostic instrument. Am J Psychiatry 127: 1653-1658.

Tzschentke TM (1998). Measuring reward with the conditioned place preference paradigm: a comprehensive review of drug effects, recent progress and new issues. Prog Neurobiol 56: 613-672.

Tzschentke TM (2007). Measuring reward with the conditioned place preference (CPP) paradigm: update of the last decade. Addict Biol 12: 227-462.

Urban NB, Kegeles LS, Slifstein M, Xu X, Martinez D, Sakr E et al (2010). Sex differences in striatal dopamine release in young adults after oral alcohol challenge: a positron emission tomography imaging study with $[(1)(1) \mathrm{C}]$ raclopride. Biol Psychiatry 68: 689-696.

Volkow ND, Wang GJ, Fowler JS, Telang F, Maynard L, Logan J et al (2004). Evidence that methylphenidate enhances the saliency of a mathematical task by increasing dopamine in the human brain. Am J Psychiatry 161: 1173-1180.

White TL, Justice AJH, De Wit H (2002). Differential subjective effects of D-amphetamine by gender, hormone levels and menstrual cycle phase. Pharmacol Biochem Behav 73: 729-741.

Wilson TD, Dunn EW (2004). Self-knowledge: its limits, value, and potential for improvement. Annu Rev Psychol 55: 493-518. 